\title{
Alter
}

Revue de phénoménologie

19 | 2011

Le langage

\section{Heidegger et la question de l'« essence » du langage}

\section{Françoise Dastur}

\section{OpenEdition}

Journals

Édition électronique

URL : http://journals.openedition.org/alter/1362

DOI : $10.4000 /$ alter.1362

ISSN : 2558-7927

\section{Éditeur :}

Association ALTER, Archives Husserl (CNRS-UMR 8547)

\section{Édition imprimée}

Date de publication : 1 octobre 2011

Pagination : 43-56

ISBN : 978-2-9522374-7-5

ISSN : 1249-8947

\section{Référence électronique}

Françoise Dastur, « Heidegger et la question de l'« essence » du langage », Alter [En ligne], 19 | 2011, mis en ligne le 01 janvier 2020, consulté le 23 janvier 2020. URL : http://journals.openedition.org/alter/ 1362 ; DOI : 10.4000/alter.1362 


\section{HEIDEGGER ET LA QUESTION DE L'«ESSENCE » DU LANGAGE}

Françoise Dastur

"Logik als Frage nach dem Wesen der Sprache ", tel est le titre que Heidegger donne à son cours du semestre d'été $1934^{1}$, cours qui a été traduit en 2008 sous le titre "La logique comme question en quête de la pleine essence $d u$ langage $»^{2}$, " pleine essence» se voulant ici la traduction du nouveau sens que Heidegger donne à partir du milieu des années 1930 au terme Wesen, qu'il ne s'agit plus d'entendre en un sens nominal comme l'essence ou la quiddité ni comme l'expression de la permanence ou de l'invariabilité d'un eidos, mais, selon le sens $\mathrm{du}$ vieux verbe wesen, comme le déploiement temporel de l'être d'une chose, comme il l'explique en 1936 dans les «Contributions à la philosophie ", où il est dit que le mot Wesen ne doit plus être compris en un sens générique, mais comme "l'avènement de la vérité de l'être » (Geschehnis der Wahrheit des Seins) ${ }^{3}$, et comme il le soulignera à nouveau en 1953 dans ses conférences de Munich sur « La question de la technique » et «Science et méditation », où il rappelle que le verbe wesen est le même mot que währen, durer, demeurer ${ }^{4}$.

De ce cours dont le manuscrit a apparemment été perdu et qui n'a pu être publié, sur la base des notes prises par les étudiants, qu'en 1998, Heidegger avait affirmé à deux reprises, une première fois dans son cours de 1951-52 sur "Qu'appelle-t-on penser? "

\footnotetext{
1. M. Heidegger, Logik als Frage nach dem Wesen der Sprache, Ga 38, Frankfurt am Main, Klostermann, 1998 (Noté par la suite Ga 38).

2. M. Heidegger, La logique comme question en quête de la pleine essence du langage, trad. F. Bernard, Paris, Gallimard, 2008. Le traducteur explique son choix de traduction pour Wesen dans l'Avant-propos.

${ }^{3}$. M. Heidegger, Beiträge zur Philosophie (Vom Ereignis), Frankfurt am Main, Klostermann, 1989, $\S 165$, p. 287.

${ }^{4}$. M. Heidegger, Essais et conférences, Paris, Gallimard, 1958, p. 41 et 55.

5. M. Heidegger, Qu'appelle-t-on penser?, Paris, PUF, 1959, p. 157.
} 
fois dans son entretien de 1954 avec un Japonais ${ }^{6}$, qu'il constitue un moment décisif, celui de la transformation de la logique en la question du Wesen der Sprache, du déploiement de l'être du langage. Cette précision permet de comprendre que ce que Heidegger nomme dans ce cours « la tâche nécessaire d'un ébranlement (Erschütterung) de la logique $»^{7}$ est une tâche qui restait encore essentiellement axée sur une certaine conception du langage, celle que l'on trouve dans Sein und Zeit, où le langage est défini comme l'Hinausgesprochenheit der Rede, l'extériorisation orale du discours ${ }^{8}$. C'est avec une telle définition du langage qui oppose l'articulation de la Rede, du discours, à l'ébruitement sonore du Sprechen, du parler, que Heidegger va être amené à rompre à partir du milieu des années 1930, car elle demeure encore sous la dépendance de la distinction d'origine husserlienne entre signification et expression, comme l'atteste une phrase du $\S 18$ dans laquelle est affirmé le caractère "fondé » (fundiert) du mot et de la parole (Wort und Sprache) par rapport aux significations (Bedeutungen) $)^{9}$.

Il n'en demeure cependant pas moins que Heidegger lui-même refuse dans une certaine mesure de considérer qu'une nette rupture $\mathrm{s}^{\prime}$ est ainsi produite, en ce qui concerne la question du langage, entre Etre et temps et la période des années trente. Dans le texte déjà mentionné de son entretien de 1954 avec un Japonais, Heidegger affirme que ses questions ont toujours tourné autour du problème du langage et de l'être et que la réflexion sur le langage a déterminé son chemin de pensée depuis le début. Il mentionne à l'appui de cette assertion le thème de sa dissertation d'habilitation de 1915 qui portait sur la doctrine des catégories et la théorie de la signification de Duns Scot, dont le nom métaphysique est grammatica speculativa ${ }^{10}$. Il admet certes que cette relation entre langage et être n'était pas à cette époque claire pour lui et il explique que c'est parce que la méditation du langage et de l'être a determiné dès le départ son chemin de pensée que la discussion de leur relation demeure le plus possible à l'arrière plan, reconnaissant pourtant qu'à cet égard «le défaut radical du livre Sein und Zeit » est qu'il « se soit trop tôt aventuré trop loin $»^{11}$. Pourtant, lorsque son interlocuteur souligne que dans Sein und Zeit, l'Erörterung, le débat au sujet du site du langage, demeure parcimonieuse, il lui conseille de « lire plus attentivement le $\S 34$ » $^{12}$,

\footnotetext{
${ }^{6}$. M. Heidegger, Unterwegs zur Sprache, Neske, Pfullingen, 1959, p. 93. (noté par la suite US) ; trad. F. Fédier, Acheminement vers la parole, Paris, Gallimard, 1976, p. 93.

7. Ga $58, \S 4$.

8. M. Heidegger, Sein und Zeit, Tübingen, Niemeyer, 1963, § 34, p. 161 (Noté par la suite SZ).

9. Ibid., p. 87.

10. US, p. 91 sq. ; trad. p. 91 sq.

11. US, p. 93 ; trad. p. 93.

12. US, p. 137 ; trad. p. 127.
} 
réitérant ainsi implicitement le jugement rétrospectif qu'il avait déjà porté sur ce paragraphe en 1946 dans la Lettre sur l'humanisme où il affirmait qu'il contient "une indication sur la Wesensdimension, la dimension d'être du langage ", car il touche à la question simple du mode d'être du langage ${ }^{13}$.

Il s'agit là d'une considération rétrospective qui constitue ce que l'on a légitimement nommé « l'auto-interprétation » de Heidegger qui comprend son propre itinéraire de pensée comme un cheminement qui connaît certes un "tournant", mais non pas une brusque interruption l'obligeant à trouver un autre point de départ. La question n'en demeure pas moins pour nous de savoir si le theme fondamental de Heidegger est, comme il semble l'affirmer en 1954, «langage et être " plus encore que " être et temps ", et s'il y a un chemin qui mène du premier thème au second, de son livre sur le temps de 1927 au recueil de ses textes tardifs sur le langage publié en 1959 sous le titre Unterwegs zur Sprache, qui fut traduit par Acheminement vers la parole, $\mathrm{du}$ fait que le terme de Sprache, nom correspondant au verbe sprechen qui signifie parler, peut effectivement être traduit indifféremment par langage ou parole, l'allemand ne connaissant pas la distinction que le français fait entre langue, langage et parole.

Il faut rappeler en effet qu'en 1927 le projet de Heidegger consistait à montrer que le temps est l'horizon transcendantal de l'être, en d'autres termes que l'être est intrinsèquement " temporal ", ce qui veut dire qu'il doit être pensé de manière verbale et en relation au temps. Ce but ne fut pourtant pas atteint dans la partie publiée de Être et temps, qui demeura un livre inachevé, car Heidegger renonça à publier la fameuse troisième section de cet ouvrage qui, par son titre "Temps et être », suggérait que la relation entre être et temps se renversait, le temps ne constituant plus seulement la dimension fondamentale de l'être de l'homme, du Dasein, mais de l'être lui-même. Cela voulait dire, précise-t-il dans la Lettre sur l'humanisme ${ }^{14}$, que « cette pensée autre qui abandonne la subjectivité » ne parvint pas à un accomplissement suffisant, car la pensée échoua "à exprimer ce renversement et n'en vint pas à bout avec l'aide de la langue de la métaphysique », ce qui laissait entendre que les vieux mots de «transcendance » et $\mathrm{d}^{\prime}$ " horizon » ne lui semblaient plus appropriés à nommer la relation entre temps et être.

Il s'avérait donc clairement qu'un autre langage que celui de la tradition était requis. Mais, comme Heidegger le souligne dans Acheminement vers la parole, la transformation requise du langage ne consiste pas en la fabrication de mots ou de suites de mots nouveaux ${ }^{15}$, comme s'il s'agissait de donner de meilleurs noms aux

13. M. Heidegger, Lettre sur l'humanisme, Paris, Aubier, 1964, p. 41.

14. Ibid., p. 69.

15. US, p. 267 ; trad. p. 256. 
choses et de changer la structure de nos énoncés afin d'exprimer plus adéquatement les phénomènes. Car si cela était le cas, cela voudrait dire que Heidegger était demeuré prisonnier de la conception logique et grammaticale du langage, de ce que les exégètes des Recherches philosophiques de Wittgenstein nomment à bon droit " l'image augustinienne du langage », c'est-à-dire une conception nominaliste du langage qui voit dans les mots seulement des noms et dans le langage un simple lexique ${ }^{16}$. La transformation du langage qui est requise est en réalité moins la transformation du langage lui-même que celle de notre relation au langage : il nous faut passer par une expérience du langage qui nous enseigne à renoncer à notre compréhension habituelle de la relation du mot à la chose comme d'une relation entre deux objets déjà tout constitués ${ }^{17}$, ce qui implique l'abandon de la conception logiciste du langage qui est demeurée dominante depuis la constitution de la logique formelle dans les écoles aristotéliciennes. Heidegger distingue en effet déjà dans son cours de 1934, entre les mots ou paroles et les termes, Worte et Wörter ${ }^{18}$, et souligne que le langage, la Sprache, " ne peut être trouvé que là où il est parlé, c'est-àdire parmi les hommes ${ }^{19}$. Par la suite, dans ses textes des années 1950, cette distinction, devenue fondamentale, permet de montrer que les paroles, contrairement aux termes, qui consistent en phonèmes et/ou graphèmes, ne sont pas des étants et ne se substituent pas aux choses qu'ils désignent, mais sont ce qui leur procure originairement la présence et l'être, de sorte que la parole ainsi considérée dans la dimension du dire est la source de l'être ${ }^{20}$.

En rompant ainsi avec ce que l'on peut nommer avec Wittgenstein la conception « pictoriale » du langage, et qui n'est rien d'autre que la conception logiciste de celui-ci, on abandonne du même coup l'opposition métaphysique que l'on a toujours établie dans la tradition occidentale entre « être » et « devenir » et qui a conduit à comprendre l'être comme quelque chose de déjà présent, de vorhanden, qu'il s'agirait par conséquent de tenter d'exprimer après coup. Dans la mesure où nous apprenons à habiter le langage au lieu de l'utiliser comme un

\footnotetext{
16. Voir L. Wittgenstein, Philosophische Untersuchungen, Suhrkamp, Frankfurt am Main, p. 13. Le début des Untersuchungen est une citation de St Augustin, Confessions, 1, 8.

17. US, p. 170 ; trad. p. 154.

18. Ga 38, § 7, p. 23.

19. Ibid., § 8, p. 24 .

${ }^{20}$. US, p. 169 ; trad. p. 153, où Heidegger définit la vocation du poète comme « la vocation au mot, entendu comme la source de l'être ». Voir aussi ce fameux passage de Qu'appelle-t-on penser?, p. 141-42: "Ce ne sont jamais de simples termes qui nous sont tout d'abord donnés", car «lorsque nous entendons ce qui est parlé, nous nous tenons dans l'espace de jeu de ce qui est parlé, où résonne la voix muette de ce qui est dit » de sorte que «Les paroles ne sont pas des termes, et en tant que telles semblables à des tonneaux ou à des seaux, d'où nous puiserions un contenu existant. Les paroles sont des sources que le dire creuse davantage, des sources qu'il faut toujours à nouveau trouver, de nouveau creuser, qui s'encombrent facilement, mais qui de temps en temps jaillissent aussi à l'improviste ».
} 
simple moyen de communication, nous quittons ce que l'on peut nommer, mais en un autre sens que celui que Derrida donne à cette expression, la métaphysique de la pré-sence, de la Vorhandenheit, c'est-à-dire de la presence conçue sur le modèle de la substance. Le dernier Heidegger donne le nom $\mathrm{d}^{\prime}$ " onto-théologie » à cette métaphysique qui conçoit l'être à la fois comme une présence subsistante et comme le fondement présent des étants au lieu de voir en lui l'avènement de l'éclaircie, de l'alètheia, qui n'advient elle-même que sur la base de la lèthè, c'est-à-dire d'un oubli ou d'une occultation abyssale. Nous nous ouvrons ainsi à une autre conception de l'être, à l'être comme Ereignis, dans la mesure où ce mot, laissé pour l'instant non traduit, nomme ce qui détermine la co-appartenance de l'être et du temps. Langage et Ereignis : ce serait là le nom du thème propre à la pensée de Heidegger, qui ne remplace pas le premier thème « Être et temps », mais l'exprime de maniere plus originelle, au sens où il rend plus explicite son problème central : le problème de la relation entre l'homme et l'être. Voilà quelle serait l'hypothèse de lecture proposée ici. Mais pour tenter de vérifier cette hypothèse, il faut commencer par retracer dans ses grandes étapes l'itinéraire du questionnement heideggérien du langage et donc repartir du $\S 34$ de Sein und Zeit.

$* * *$

Dans ce paragraphe, intitulé "Dasein et discours. Le langage ", le discours (Rede) est compris comme un élément constitutif de l'être dans le monde, c'est-à-dire comme un mode spécifiquement humain de l'être. À cet égard, Heidegger n'hésite pas à s'appuyer sur la définition grecque de l'homme comme zôon logon ekhon, qu'il considère pourtant à d'autres égards comme insuffisante. Cette définition ne signifie cependant pas pour lui que l'homme est un être capable de parler au sens de produire des sons vocaux, mais plutôt un être capable d'ouverture à soi et au monde. Pour Heidegger la relation sujet-objet est dérivée, car ce qui permet à un sujet de rencontrer un objet, c'est l'établissement préalable d'un terrain commun entre eux. Le monde est ce terrain commun : il précède l'objet, car il est une structure du Dasein, et non pas la totalité de l'étant. Le monde n'est ni la somme des objets ni une structure du sujet, il n'est ni purement objectif, ni purement subjectif, il est cette structure de Bedeutsamkeit, de significativité, à partir de laquelle il peut y avoir rapport intentionnel entre sujet et objet, et ce que Heidegger laisse entendre dans Sein und Zeit, c'est que l'ouverture au monde du Dasein ne consiste précisément pas en un acte intellectuel, mais relève plutôt de ce que la tradition nomme " affectivité ». À cet égard, l'existential que Heidegger nomme Befindlichkeit, disposition, a une importance fonda- 
mentale ${ }^{21}$ : c'est le fait de «se trouver» et "de se sentir » dans le monde, selon le double sens, passif et actif, du verbe pronominal sich befinden, qui caractérise originellement l'existant qui ne pourra qu'ensuite rencontrer sur cette base l'étant individuel. Cette ouverture au monde qu'il est toujours-déjà constitue le véritable sens du "passé » pour le Dasein, mais c'est un "passé » qui n'est pas révolu, qui ne constitue nullement un fond substantiel pour lui, mais qui au contraire s'actualise sans cesse en étant repris par la compréhension, qui est projection du monde. On peut certes penser que Befindlichkeit et Verstehen ne sont que les nouveaux noms de ces sources séparées de la connaissance que sont chez Kant la sensibilité et l'entendement, mais ce qui est précisément propre à Heidegger, c'est le fait de penser leur « cooriginarité » et donc leur entrelacement, ce qui implique du point de vue temporel que passé et avenir, être-jeté et projet, passivité et activité, sont inséparables et renvoient l'un à l'autre.

Or, à ces deux existentiaux, Heidegger ajoute l'existential du discours 22 , Rede, qui assure donc l'actualisation des deux premiers ou leur articulation. Car il n'est pas d'existence qui ne s'explicite en un discours sur soi et le monde. Mais il ne faut pas confondre le discours en tant que structure existentiale et l'énonciation (Aussage), qui en est un mode dérivé et qui, lui, correspond à l'attitude proprement théorique et à l'ontologie de la Vorhandenheit, de la présence donnée. Il y a en effet une explicitation plus originelle que l'enonciation et qui rend possible cette dernière. L'explicitation, dans laquelle Heidegger voit le développement de la compréhension, c'est-à-dire sa realisation concrète, est caractérisée par la structure de l'en tant que. Elle peut s'effectuer par l'action ou par le discours. Ce qui est remarquable à ce niveau, c'est que la structure d'explicitation de l'étant maniable (du Zuhandene) nous renvoie à ses capacités, à ce que Platon nomme ses dunamai, sans que soit posé à part le sujet des dunamai, car l'outil, le Zeug, se confond avec elles: le marteau est « compris » comme servant à marteler, le clou comme servant à accrocher, etc. L'explicitation peut donc ici se réaliser hors langage, dans l'antéprédicatif. Ce qu'apporte de nouveau l'énonciation, c'est-à-dire la prédication, c'est la position du sujet, du substrat. Or, pour cela, il faut un changement, une modification du rapport au monde, qui nous fait passer de l'outil à la chose pure et simple. Ce changement n'est cependant pas une rupture totale avec la préoccupation, qui demeure le mode fondamental de notre rapport au monde, mais une modification de celle-ci, une modification de l'intérêt, pour reprendre ici une terminologie kantienne, qui de pratique devient théorique. La préoccupation se

21. Cf. SZ, § 29 .

22. Ibid., $\S 28$, p. 133. 
réduit alors au pur regard, isole l'étant particulier, le pose sous la figure du substrat, du sujet auquel s'ajouteraient des qualités.

Ce qui s'ouvre dès lors, c'est l'espace prédicatif, logique au sens aristotélicien du terme. Or ce niveau est précisément celui du rapport à l'objet et de l'intentionnalité de la conscience. Il y a là un renversement des rapports qui régissent la quotidienneté. C'étaient ses capacités et sa destination qui constituaient l'explicitation de l'outil, alors que maintenant, c'est la substance qui constitue la raison de ses prédicats. Un tel renversement a un retentissement sur la conception du langage : le langage est identifié à l'énonciation et l'énonciation elle-même est considérée comme une présence donnée. C'est pourquoi la langue est comprise dans la tradition philosophique, et déjà chez les Grecs tardifs, à partir de la phônè, de la présence donnée du son, de sa "matérialité ", de sorte que toute theorie du langage est d'abord phono-logie. Heidegger montre que c'est d'une telle conception du langage comme ensemble d'éléments donnés et de la langue comme substance que provient la théorie classique de la vérité comme adéquation de la res à l'intellect, en tant que l'un et l'autre constituent deux étants qu'il faut donc supposer homogènes et de même valence ontologique.

Pourtant logos ne signifie pas originellement «langage », mais "discours » et a le sens de rendre manifeste. En 1927 Heidegger met l'accent sur le fait que "les Grecs n'ont pas de mot pour langage ", parce que, explique-t-il, "ils ont "de prime abord" compris ce phénomène comme discours $»^{23}$. Il ne faut donc pas traduire le mot Rede par parole comme le fait François Vezin dans sa traduction de Sein und Zeit. A cet égard, la traduction qu'en donne de son côté Emmanuel Martineau n'est pas plus éclairante, car il rend Rede par "parler » et Sprache par " parole », là où François Vezin les traduit respectivement par « parole » et « langue ». Il semble en effet que la traduction proposée par les premiers traducteurs de Sein und Zeit, Boehm et de Waelhens, de Rede par discours et Sprache par langage, convienne mieux. Heidegger comprend vraisemblablement le mot Rede à partir de son étymologie qui le rattache à la racine indo-européenne *ar, qui signifie joindre, articuler ensemble, ce que, dans une certaine mesure, le mot discours, qui implique une succession d'éléments articulés les uns aux autres, peut exprimer. Il le définit en effet comme « l'articulation de ce qui est compréhensible $\gg^{24}$, mais cela ne signifie cependant pas qu'il vienne après la compréhension comme une sorte de post-structuration de ce qui a déjà été compris. Au contraire la compréhension est déjà en elle-même articulée, de même que la disposition, la Befindlichkeit, implique toujours une certaine compré-

23. Ibid., § 34, p. 165.

24. Ibid., §34, p. 161. 
hension : les trois existentiaux de l'ouverture du Dasein (compréhension, disposition et discours) sont co-originels et non pas "fondés " les uns sur les autres. Par contre, le langage (Sprache) n'est pas dans Etre et temps un phénomène originel, mais un phénomène fondé dont la fondation existentiale-ontologique peut être trouvée dans le discours (Rede). On peut maintenant comprendre pourquoi le phénomène du langage a déjà pu être analysé sous la forme de l'énoncé (Aussage) dans le $\S 33$, c'est-à-dire avant que le langage ne devienne un thème : l'énoncé ne peut être considéré comme une "dérivation extrême » de l'explicitation que parce que l'explicitation, qui n'est rien d'autre que le développement de la compréhension et l'appropriation de ce qui a été compris, n'est elle-même possible que dans sa relation au discours ${ }^{25}$.

Le discours est ainsi la condition de possibilité du langage et le langage l'être mondain du discours, son extériorisation orale, de sorte qu'il faut dire que «les significations se muent en paroles » et non pas, à l'inverse, que "des mots-choses viennent à se pourvoir d'un sens $»^{26}$. Le langage est donc découvert à l'intérieur du monde comme s'il était un étant maniable, c'est-à-dire un simple instrument, tandis que le discours, qui peut demeurer non formulé, possède les possibilités existentiales du faire silence et de l'entendre. L'entendre ne peut en effet pas plus être identifié à la perception acoustique que le faire silence ne peut être identifié au mutisme. Car la perception de purs sons n'est possible que sur la base de l'entendre, tout comme la parole n'est possible que sur la base du discours, parce qu'entendre signifie toujours compréhension d'un étant maniable au sein du monde et non pas l'interprétation des sensations internes d'un sujet ayant seulement une relation médiate avec le monde. Faire silence ne signifie pas que la capacité de parler fasse défaut, mais présuppose au contraire la possibilité de dire, c'est-à-dire de montrer, de sorte que le silence est ce mode originel du discours qui peut même faire comprendre mieux que la parole elle-même. Il ne faut donc pas confondre les éléments constitutifs du discours avec ceux de la parole et du langage : les premiers sont des caractères existentiaux, les seconds des caractères ontiques.

Mais après cette brève récapitulation de l'analyse du discours dans le §34, la question demeure : comment Heidegger a-t-il pu rétrospectivement trouver dans cette section, où n'est reconnue au langage qu'une position subordonnée, une «indication sur la Wesensdimension, la dimension d'être du langage ${ }^{27}$ ? C'est en fait seulement à la fin du $\S 34$ que Heidegger pose la question du mode d'être du

\footnotetext{
25. Ibid., § 34, p. 160 et 161.

26. Ibid., § 34, p. 161. On reprend ici l'excellente traduction de ce passage par R. Boehm et A. de Waelhens dans L'Etre et le Temps, Paris, Gallimard, 1964.

27. Lettre sur l'humanisme, p. 41.
} 
langage. Parce que les mots prononcés ou écrits sont découverts à l'intérieur du monde, ils peuvent être considérés comme des choses maniables, de sorte que le langage semble n'être qu'un simple instrument de communication. Mais la maniabilité et l'instrumentalité sontelles le mode propre de l'être du langage et le langage n'est-il pas plutôt un mode d'être du Dasein, un élément existential semblable au discours, et non pas seulement un phénomène existentiel et ontique ? Ou bien le langage n'a-t-il ni le mode d'être d'un instrument ni le mode d'être du Dasein ? Dans ce dernier cas, le langage ne pourrait pas être inclus parmi les différents modes d'être, mais il devrait être référé à l'être lui-même dans son unité. Heidegger ne développe pas, comme on le fait ici, les implications de cette question, mais il n'en déclare finalement pas moins que, parce que l'objet de la science du langage demeure obscur et ontologiquement non décidé, le temps est venu pour la philosophie de cesser de considérer le langage comme le domaine particulier de la "philosophie du langage» afin de commencer à questionner les "choses mêmes », ce qui veut dire : laisser le phénomene du langage se montrer en soi-même ${ }^{28}$.

$* * *$

Par cette relecture du $\S 34$ de l'œuvre de 1927, nous avons pu mesurer, en dépit du peu d'indications relatives au phénomène du langage qu'il contient, l'importance dévolue au discours, qui n'est pas seulement une structure existentiale spécifique, mais une structure de l'existence qui se rapporte au Dasein dans sa totalité. Le langage luimême, et non pas seulement le discours, ne peut devenir un thème essentiel que s'il n'est plus compris comme l'énonciation phonétique des mots, mais, à l'instar du discours, comme un montrer, c'est-à-dire un dire. Dans le $\S 7$ de Etre et temps, Heidegger, à la suite d'Aristote, comprenait le logos grec comme discours et lui donnait le sens de manifestation de ce qui est en question dans le discours ${ }^{29}$. Dans la période qui suit, Heidegger approfondit son analyse du logos et entreprend $\mathrm{d}^{\prime}$ " ébranler » la logique traditionnelle afin de reconduire le logos à son fondement, c'est-à-dire à son sens initial. C'est ainsi qu'il va être conduit à penser le langage lui-même non plus comme un processus purement phonétique d'expression et de communication - ce qui est en fait la conception métaphysique traditionnelle du langage qui est encore celle qui, en un certain sens, prévaut dans $\hat{E}$ tre et temps - mais comme une monstration, c'est-à-dire comme l'événement même de l'éclaircie. Comme il le déclare en 1946 à Jean Beaufret, le langage ne peut être pensé conformément à son essence à

${ }^{28 .}$ SZ, § 34, p. 166.

29. Ibid., § 7 B, p. 32. 
partir de son caractère de signe et de signification, et pas davantage comme "l'expression d'un être vivant», car il est « la venue à la fois éclaircissante et celante de l'être lui-même $»^{30}$.

Dans la seconde des trois conférences qu'il donne en 1957 sous le titre à nouveau de "Das Wesen der Sprache », "Le déploiement de la parole », Heidegger, commentant ce vers de Stefan George qui dit "Aucune chose ne soit, là où le mot fait défaut », souligne que c'est précisément ce rapport entre la chose et le mot qui a, au matin de la pensée occidentale, assailli la pensée de manière si stupéfiante qu'il s'est annoncé par un seul mot, celui de logos, qui est en même temps le nom de l'être et celui du dire ${ }^{31}$. Dans les cours qu'il a consacrés au début des années quarante aux Présocratiques et en particulier à Héraclite, Heidegger avait en effet entrepris de remonter a un sens du logos plus initial que celui que l'on trouve chez Aristote. Ce qui advint au commencement de la pensée occidentale avec Héraclite, c'est, expliquait-il déjà, avec le mot directeur de logos, l'expérience de l'être du langage comme "pose recueillante », langage voulant alors dire « rassembler ce qui est présent et le laisser étendu-devant dans sa présence $»^{32}$. Mais, ajoutait-il, "Les Grecs habitaient dans cet être du langage. Seulement ils ne l'ont jamais pensé, et pas même Héraclite ». C'est pourquoi lorsqu'ils ont voulu definir l'essence de la langue, ils l'ont d'abord comprise comme une expression fondée sur un processus phonétique. Le langage a par conséquent été défini par Aristote comme phonè semantikè, comme un son qui signifie, et depuis le langage a constamment été envisagé sous cet angle, comme le montre encore le titre que Husserl a donné à la première de ses Recherches logiques : "Expression et signification». La supériorité de la langue grecque, qui a été soulignée par Heidegger dès 1935, dans son cours sur l'Introduction à la métaphysique33, et par la suite dans sa conférence de 1955 à Cerisy intitulée Qu'est-ce que la philosophie ? ${ }^{34}$, n'a en réalité rien à voir avec une qualité spéciale qui n'appartiendrait qu'à elle grecque, et qui la rendrait plus apte qu'aucune autre langue à dire l'être. Il faudrait rappeler à cet égard l'affirmation de Heidegger dans "La parole d'Anaximandre », texte tiré d'un essai écrit en 1946: "L'être parle partout et toujours au travers de toute langue. La difficulté n'est pas tellement de trouver, dans la pensée, le mot de l'être, mais bien plutôt de bien maintenir le mot trouvé dans la retenue d'une pensée véritable »35. La supériorité de la langue grecque vient simplement du fait que les Grecs

\footnotetext{
30. Lettre sur l'humanisme, p. 65.

31. US, p. 185 ; trad. p. 169.

32. «Logos », Essais et conférences, Paris, Gallimard, 1958, p. 276 (Conférence de 1951 reprenant un thème développé en 1944 dans un cours consacré à Héraclite).

33. M. Heidegger, Introduction à la métaphysique, Paris, PUF, 1958, p. 67.

34. M. Heidegger, Questions II, Paris, Gallimard, 1968, p. 20.

35. M. Heidegger, Chemins qui ne mènent nulle part, Paris, Gallimard, 1980, p. 442.
} 
habitaient leur langue, ce qui veut dire que, par l'intermédiaire des mots grecs, ils étaient immédiatement mis en relation avec les «choses mêmes » et non pas captifs d'un royaume de signes arbitraires. Ils ne possédaient pas une langue supérieure à d'autres, mais ils avaient un rapport différent à leur langue, un rapport d'habitation, et non une relation simplement instrumentale. C'est la raison pour laquelle ils inventèrent la philosophie, parce qu'habiter leur langue ne signifiait pas pour eux être dans la familiarité avec leur idiome propre et le maîtriser entièrement, mais au contraire être ouvert à son étrangeté et se voir forcé de se l'approprier, c'est-à-dire, comme le souligne Heidegger dans son cours sur Parménide de 1942-43, de traduire le grec en grec, comme c'est toujours le cas pour chaque penseur à l'égard de sa propre langue ${ }^{36}$. Car, comme Heidegger l'explique dans la Lettre sur l'humanisme, l'habitation authentique, qui se dit en grec èthos, ne signifie pas seulement la familiarité avec le monde qu'on habite, elle requiert aussi et surtout la capacité d'apercevoir le non familier (das Ungeheure) dans le familier (im Geheuren), le daimôn dans l'anthrôpon, en $\mathrm{d}^{\prime}$ autres termes dieu en l'homme et l'étranger dans $\mathrm{l}^{\prime} \mathrm{ami}^{37}$. Habiter veut dire en effet être capable de maintenir la distance au sein de la proximité et de faire place à l'étrangeté (die Unheimlichkeit) dans son propre lieu natal (in der Heimat).

Mais en suivant les Grecs qui ont, sans cependant le penser, montré que le langage est une dimension de l'être, Heidegger est amené à renverser complètement la détermination qui a d'abord été donnée du langage dans la tradition occidentale : alors que l'homme s'est jusqu'ici comporté comme s'il était le créateur et le maître du langage, il semble que ce soit maintenant au contraire ce dernier qui le régente ${ }^{38}$. La relation instrumentale que l'homme métaphysique entretient avec le langage semble ainsi s'être diamétralement inversée, au sens où maintenant, c'est le langage qui «a besoin » de $l^{\prime}$ homme et qui $l^{\prime}$ " emploie » (braucht) à son service ${ }^{39}$. Un tel renversement du point de vue métaphysique ne pourrait pourtant que conduire, comme Heidegger le souligne lui-même à propos de l'existentialisme sartrien dans la Lettre sur l'humanisme ${ }^{40}$, à une nouvelle forme de métaphysique, et non à sa destitution. Cela signifierait donc le retour des fantômes de la métaphysique sous la forme d'un langage complètement séparé de la parole humaine et représenté par conséquent sous la forme d'une entité fantasmatique et existant en soi qui ne peut être rencontrée tant que notre méditation sur le langage

\footnotetext{
36. M. Heidegger, Parmenides, Ga 54, Frankfurt am Main, Klostermann, 1992, p. 17.

37. Lettre sur l'humanisme, p. 151.

38. C'est ce que Heidegger semble affirmer dans sa conférence de 1951 "Bâtir, habiter, penser ", Essais et conférences, p. 172.

39. US, p. 260 ; trad. p. 249.

40. Lettre sur l'humanisme, p. 69-71.
} 
demeure dans la sobriétét ${ }^{1}$. Le risque du retour de la métaphysique est clairement impliqué dans les énoncés tautologiques par lesquels Heidegger énonce la nouvelle essence du langage : «le langage parle $»^{42}$, « le langage est le langage $»^{43}$. Il l'est aussi par le but qu'il se donne à lui-même dans Acheminement vers la parole : réfléchir sur le langage lui-même et uniquement sur lui, parce que "le langage luimême est langage et rien de plus $»^{44}$. La tentative de porter à l'évidence le fait que le langage n'a pas d'autre fondement que luimême pourrait mener à une idéalisation du langage, à une sorte de platonisme d'un nouveau genre. C'est là en fait un risque impliqué dans le mode phénoménologique de penser, c'est-à-dire le mode grec de penser, et le reproche de "réalisme des essences" adressé à Husserl après la publication des Recherches logiques le faisait clairement apparaître ${ }^{45}$. Mais si l'essence husserlienne n'est pas une « hypostase » platonicienne puisqu'elle n'existe pas au même titre que les choses, comme Platon lui-même l'affirmait d'ailleurs déjà, cela ne signifie pourtant pas que Husserl ne demeure pas authentiquement platonicien en un sens plus profond dans son mode de pensée eidétique. Heidegger rompt, quant à lui, plus décisivement avec le mode de pensée platonicien et se situe de cette manière "au-delà » des Grecs et de la manière dont ils ont compris la philosophie, à savoir comme une affaire de vision, comme il n'hésite pas à l'affirmer dans une de ses dernières conférences ${ }^{46}$. C'est pourquoi lorsque, après avoir montré que pour penser l'essence du langage, il est nécessaire que nous nous mettions d'abord à l'écoute du langage au sein duquel nous nous mouvons toujours déjà ${ }^{47}$, il entreprend de renverser l'expression "Wesen der Sprache » en "Sprache des Wesens », il prend soin de souligner que le mot Wesen n'est pas pris dans le même sens dans les deux expressions, car dans la première il a le sens traditionnel de quiddité, essentia, alors que dans la seconde il est pris au sens verbal $d$ 'un être qui se déploie et dure ${ }^{48}$. Ce que Heidegger nomme alors «das Wesen der Sprache » ne signifie plus «l'essence du langage », comprise comme le «visage » non historique qu'il montre à l'homme et dont celui-ci, en tant que mortel, est par conséquent séparé, mais "l'événement du langage », le déploiement de son être en tant qu'il requiert la parole humaine. C'est donc déjà cette trans-

\footnotetext{
41. US, p. 255-256 ; trad. p. 242.

42. Ibid., p. 12, 20, 254 ; trad. p. 15, 22, 241.

43. Ibid., p. $12-13$; trad. p. 14.

44. Ibid.

45. Cf. E. Husserl, Idées directrices pour une phénoménologie, Paris, Gallimard, 1950, § 22, «Le reproche de réalisme platonicien. Essence et concept ».

46. M. Heidegger, "La fin de la philosophie et la tâche de la pensée » (1968), Questions IV, Paris, Gallimard, 1976, p. 137.

47. US, p. 176 et 180 ; trad. p. 160 et 165.

48. Ibid., p. 201 ; trad p. 186.
} 
formation de la compréhension de ce qu'est le Wesen qui est en ellemême la pensée de l'Ereignis.

Nous savons maintenant, depuis la publication des Beiträge zur Philosophie ${ }^{49}$, que, sous le nom d'Ereignis, Heidegger a développé dès $1936^{50}$ une nouvelle conception de l'être, qui n'est plus considéré comme le fondement des etants, mais comme l'événement, à partir d'une occultation abyssale, d'une éclaircie qui n'est pas le fait de l'homme, mais dans laquelle celui-ci se tient. Dans la perspective de cette nouvelle conception, l'homme n'est plus considéré comme le fondement jeté de l'éclaircie, mais comme celui qui demeure exposé à elle et qui lui est redevable de son propre être. Alors qu'en 1927 l'éclaircie de l'être provenait de la projection et de la transcendance de l'être de l'homme, elle est maintenant comprise comme cette clairière dans laquelle l'homme se tient et à l'adresse (Anspruch) de laquelle il a à répondre et à correspondre (ent-sprechen). Le Verhältnis, le rapport de l'être à l'homme, ne doit pas être compris comme une simple relation entre deux entités séparées, mais comme la manière selon laquelle l'être se retire lui-même en laissant être le Da-sein. C'est dans la conférence qu'il donne en 1957 sur «Le principe d'identité » que Heidegger nomme Er-eignis, co-propriation, cette co-appartenance de l'être et de l'homme, qui n'est ni coïncidence, ni relation dialectique, mais être l'un pour l'autre et con-stellation de l'homme et de l'être ${ }^{51}$. Il y explique que l'Ereignis ne doit pas être considéré comme un événement advenant dans le monde, comme pourrait le laisser penser le sens ordinaire de ce mot, mais comme singulare tantum, comme ce qui advient de manière unique et qui n'est autre que l'ouverture de la clairière de la manifestation des étants et de leur venue à leur propre (Eigen). Mais cet Er-eigen, cette propriation, n'est pas un processus qui prend place de lui-même, il requiert au contraire de manière essentielle la participation de l'homme. La propriation doit donc être comprise, en accord avec la véritable étymologie du mot Ereignis, qui renvoie non pas au mot eigen (propre) mais au mot Auge (œil), comme l'appel du regard de l'être en direction de l'homme : das Ereignis eraügt den Menschen - l'Ereignis appelle l'homme du regard 52 .

C'est dans la conférence intitulée "Le chemin vers le langage » qu'il prononce en 1959 que Heidegger déclare que l'Ereignis «octroie aux mortels le séjour dans le déploiement de leur être, leur Wesen, de sorte qu'ils soient capables d'être ceux qui parlent ${ }^{53}$. L'Ereignis se déploie donc comme langage. C'est à partir de là qu'il est possible de

\footnotetext{
49. Les Beiträge zur Philosophie (Vom Ereignis), texte écrit par Heidegger entre 1936 et 1938, ont été publiés en 1989, pour le centième anniversaire de la naissance de Heidegger.

${ }^{50}$. Cf. US, p. 260, note 1 ; trad. p. 248 , note 19.

51. M. Heidegger, «Identité et différence », Questions I, Paris, Gallimard, 1968, p. 267 et 270-271.

52. Ibid.

53. US, p. 259 ; trad. p. 149.
} 
comprendre que le langage n'est pas une simple capacité humaine. Certes le langage a été représenté depuis longtemps à partir du parler compris comme phénomène corporel et sensible, comme l'attestent les termes de glossa, lingua, language, langue, et celui de Mundart, mot qui veut dire dialecte en allemand et signifie littéralement " manière de bouche ». Il ne s'agit pourtant pas pour Heidegger d'opposer la langue à la parole, comme le fait Saussure, et de considérer comme secondaire l'ébruitement vocal, mais de se demander s'il suffit d'expliquer de manière physiologique ce qui, dans le phénomène du langage, relève de la corporéité et si on peut le réduire au domaine métaphysique du sensible en tant qu'opposé à l'intelligible ${ }^{54}$. Ce qui constitue à ses yeux le propre de la voix, c'est son appartenance à la terre, comme Hölderlin l'a compris, lui qui nous suggère de voir dans le langage «la fleur de la bouche », en laquelle "fleurit la terre à la rencontre de la floraison du ciel $»^{55}$. Ce qui résonne dans la voix et constitue l'élément terrestre du langage doit donc être compris à partir de l'entre-appartenance de la terre et du ciel, lesquels ne sont plus opposés comme le sensible et l'intelligible, mais renvoient au contraire l'un à l'autre comme l'ouvert (du ciel) qui requiert pour apparaître l'obscurité de ce qui se retire en soi (la terre). L'événement par lequel s'ouvre ce que Heidegger nomme le Geviert, le Cadre du monde, à savoir le vis-à-vis de la terre et du ciel, de Dieu et de l'homme, est en lui-même silencieux, mais c'est précisément à cet Ereignis der Stille que les mortels que nous sommes appartenons ${ }^{56}$. Car $c^{\prime}$ est en tant que mortels, c'est-à-dire capables de faire l'expérience de la mort, et seulement en tant que tels, qu'il nous incombe d'y répondre et de le faire résonner dans notre voix. C'est donc ce Gelaüt der Stille, cette résonance du silence, qui fait du langage humain die Sprache des Wesens, le langage du déploiement de l'être ${ }^{57}$.

Ici, souligne Heidegger, "le rapport entre la mort et le langage jaillit tel un éclair, mais il est encore impensé ${ }^{58}$. Ce qui demeure en effet encore à penser, c'est que la mort en tant qu'abri de l'être et source nocturne de toute lumière est ce qui accorde au monde de régner et à l'homme d'exister et de parler. Car c'est pour ainsi dire sur ses lèvres mêmes que, comme le dit Hölderlin, " des mots, comme des fleurs, viennent à naître ${ }^{59}$, paroles dont l'éclosion sonore fait apparaître, plus qu'elle ne le brise, l'immense silence dont elle est née.

\footnotetext{
54. Ibid., p. 204 ; trad. p. 190.

55. Ibid., p. 206 ; trad. p. 191

56. Ibid., p. 214 ; trad. p. 200.

57. Ibid., p. 216 ; trad. p. 202.

58. Ibid., p. 215 ; trad. p. 201.

59. Hölderlin, « Pain et vin » (5e strophe), CEuvres, Paris, Gallimard, 1967, p. 811.
} 